\title{
Large Rabi splitting of mixed plasmon-exciton states in small plasmonic moiré cavities
}

\author{
Simge Ates, ${ }^{1}$ Ertugrul Karademir,,${ }^{1}$ Sinan Balci, ${ }^{2}$ (1) Coskun Kocabas,${ }^{1}$ and \\ Atilla AydinLI ${ }^{1, *}$ (1) \\ ${ }^{1}$ Department of Physics, Advanced Research Laboratories, Bilkent University, 06800 Ankara, Turkey \\ ${ }^{2}$ Department of Photonics, Izmir Institute of Technology, 35430 Izmir, Turkey \\ ${ }^{*}$ Corresponding author: akareakare@gmail.com
}

Received 12 August 2020; revised 10 September 2020; accepted 10 September 2020; posted 14 September 2020 (Doc. ID 405278); published 13 October 2020

\begin{abstract}
We report on exciton-plasmon coupling in metallic moiré cavities, both numerically and experimentally. Moiré cavities fabricated using double exposure laser interference lithography were filled with a molecular dye, J-aggregate. Polarization-dependent spectroscopic reflection measurements supported by simulations reveal strong coupling of organic dye excitons with cavity modes of the plasmonic moiré cavities. An anti-crossing at zero detuning, a clear indication of strong coupling, has been observed when the excitonic absorption band resonates with the cavity mode. Large Rabi splitting energies owing to the strong coupling of plasmons and excitons are clearly observed. () 2020 Optical Society of America
\end{abstract}

https://doi.org/10.1364/OL.405278

Interactions between a quantum mechanical system and an electromagnetic field have recently received a great amount of interest [1]. They can form a hybrid state due to the coupling of optical modes to two or several level quantum systems providing for interesting physics and applications [2]. Excitonic sources such as dyes [3], self-assembled and aggregated chains of cyanine dyes (J-aggregates) $[4,5]$ and quantum dots [6] can be considered as examples of two-level quantum mechanical systems. Electromagnetic fields can be provided by various photonic sources, including, for example, surface plasmon polaritons (SPPs). As expected, strong interaction of such excitonic systems with SPPs leads to large Rabi splitting energies ranging from a few $\mathrm{meV}$ to a few $100 \mathrm{meVs}$ [7]. When the rate of energy transfer between excitons and the plasmonic modes are larger than the decay rate of individual states, a strong coupling regime between the two states is realized $[5,8]$. Achieving the conditions for strong coupling requires an increase of the oscillator strength of the excitonic system, as well as decreasing plasmonic losses. Providing a photonic cavity for feedback increases strong coupling as well. Such cavities have been constructed using various approaches such as photonic bandgap cavities and Bragg cavities [8-10]. Lidzey et al. showed the coupling of two different excitonic modes via photon mediation in organic semiconductor microcavities [9].
In addition, they demonstrated the strong coupling of excitons to the optical modes of organic microcavities, even at room temperature [8]. The strong coupling of a single quantum dot to a photonic inorganic nanocavity has also been studied, and Rabi splitting has been observed in the dispersion spectra [11]. Furthermore, plasmonic picocavities, which are indeed smaller than $1 \mathrm{~nm}^{3}$, have been used to boost light matter interaction [12].

The strong coupling of nanoparticle mediated localized SPPs and excitons in organic semiconductor J-aggregates has been studied both theoretically and experimentally, which shows larger Rabi splitting energies than previous studies, and even ultrastrong coupling regime has been reached $[1,13,14]$. Unlike most quantum dots with blinking problems, J-aggregates provide excitons with strong oscillator strength as attested by their very narrow absorption peak in the red region of the electromagnetic spectrum. However, the strong interaction of excitons with plasmonic modes of metallic cavities has been less studied. For example, the effect of plasmonic cavity size on the coupling strength in plasmon-exciton mixed states has not yet been studied. Here we report on the interaction of J-aggregates with propagating SPPs on corrugated metallic surfaces and moiré cavities. We show that small plasmonic cavities demonstrate large plasmon-exciton interactions due to the small mode volume of the cavities. Unlike SPPs on flat metallic surfaces, confinement in a moiré cavity leads to slow plasmon polaritons that should enhance the light-matter interactions at nanoscale dimension [15]. The metallic moiré surfaces have been fabricated by double exposure laser interference lithography. The $\mathrm{J}$-aggregate in a polymer layer has been placed inside the plasmonic moiré cavities. Furthermore, we solve the wave equation with a finite difference approach for various corrugated surfaces to determine the dispersion of plasmonic modes and introduce the excitons on the corrugated surfaces as absorbing layers through a complex refractive index with adjustable oscillator strength. We observe that the cavity plasmons interact strongly with the excitons and, hence, the dispersion curve of the hybrid system exhibits a characteristic anticrossing of the cavity plasmons and J-aggregate excitons. The results indicate that small plasmonic cavities show large Rabi splitting energies owing to the small mode volume of small plasmonic coupled cavities. 


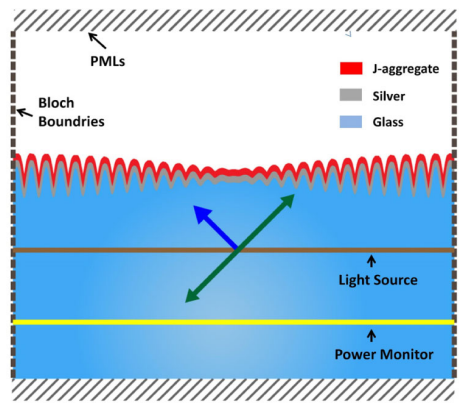

Fig. 1. Schematic view of the simulation model for excitonplasmon coupling on moiré surfaces. The surfaces are patterned to obtain a moiré cavity by using the function $\left(\sin \left(2 \pi x / \lambda_{1}\right)+\right.$ $\left.\sin \left(2 \pi x / \lambda_{2}\right)\right)$, where $\lambda_{1}$ and $\lambda_{2}$ are slightly different periods of sine functions.

The theoretical results agree rather well with the experimentally obtained results.

Numerical solutions for the plasmonic modes on a metal surface are well known. A period of the corrugated surface or a moiré cavity is introduced in the computational window. We employ the central difference finite difference approach with periodic boundary conditions for a realistic description of the experiments. A schematic view of the simulation window for exciton-plasmon coupling on a moiré surface is given in Fig. 1. Generally, a soda-lime glass was used as the substrate in the experiments. Thin Ag film coating supporting propagation of SPPs was added onto the glass substrate. On top of the plasmonic layer, an absorbing dielectric layer consisting of a thin polymer film with the exciton source was located. Optical properties of J-aggregate dye molecules were used as the exciton source, which was modeled as a Lorentz absorber. Absorbing boundaries in the form of perfectly matching layers were used in the direction parallel to the plasmonic surface. Lateral boundaries of the simulation window are periodic Bloch boundaries due to the periodic nature of the structures studied in the experiments. A tunable plane wave source was used as the light source. A power monitor measured the total electromagnetic power that passed through a specified region; for $2 \mathrm{D}$, a line was placed below the light source. The output of the power was then normalized with respect to the total power of the source. Corrugated surfaces are modeled with a sine function to obtain uniform gratings, while moiré surfaces are expressed by $S(x)=\cos (2 \pi x / D) \sin (2 \pi x / d)$, where $D=\left(\Lambda_{1} \Lambda_{2} / \Lambda_{1}+\Lambda_{2}\right), \quad$ and $\quad d=\left(\Lambda_{1} \Lambda_{2} / \Lambda_{1}-\Lambda_{2}\right) ; \quad \Lambda_{1}$ and $\Lambda_{2}$ are the periods of the superimposed uniform gratings. Uniform periodicity of the structure is represented by $d$, while $D$ stands for the cavity size or the periodicity of the envelope function of the cavity [16]. Remarkably, the size of the plasmonic cavity on moiré surfaces; thus, the optical properties of the cavity mode can be tuned flexibly. Reflection curves were obtained for incidence angles of 0.2 deg intervals. In fact, strong coupling of incident light to surface plasmons requires matching of incident light and plasmon momenta. Thus, for each wavelength corresponding to a different energy, we scan the incidence angle until we reach the critical angle where the coupling takes place. Hence, by plotting wavelength versus the angle of incidence, the response of the surface reflectivity in a heat map, dispersion of the SPPs was indeed obtained.
For the experiments, moiré patterns were fabricated by double exposure laser interference lithography [16] on glass substrates, as reported in our previous works [15,16]. A $40 \mathrm{~nm}$ thick Ag thin film was evaporated on the patterned surfaces to support propagation of SPPs. Aggregated TDBC (cyanine dye (5,5,6,6'-tetrachloro-di-(4-sulfobutyl) benzimidazolo carbocyanine) molecules (J-aggregate) were used as excitonic matter component, and metal-coated surfaces were used to support propagation of SPPs. A polyvinyl alcohol (PVA) polymer in water (1.5\% PVA solution) was used for the TDBC molecules to disperse in PVA matrix, which was spin-coated (3000 rpm) on the metal surfaces [5]. The periodicity of the cavities was controlled by tuning the periods of sine functions that are superimposed to form the cavities $[15,16]$. In reflection measurements, a Kretschmann configuration, where the Ag-coated sample was attached to a prism with an index matching fluid, eliminating reflection losses associated with a glass-air interface was used. A tunable monochromatic light source was used to scan the frequency response of the whole hybrid structure, while momentum of the incoming light was tuned by varying the incidence angle. The incident light is p-polarized (transverse magnetic $[\mathrm{TM}]$ ), and all the reflection measurements were normalized with the s-polarized incident light (transverse electric [TE]). Note that SPPs are polarization sensitive; thus, they can be only excited by p-polarized incident light.

In order to experimentally and theoretically investigate exciton-plasmon coupling in plasmonic cavities, metallic moiré surfaces were used. Simulations were performed on moiré surfaces as a function of the cavity size. It should be noted here that as the cavity size increases on moiré surface localization of SPP increases, and the Q-factor of the cavity, the energy stored in the bare plasmonic cavity divided by energy lost per cycle, increases [16]. Here the coupled periodic nature of interference lithograph, is accommodated by the application of the periodic boundary conditions in the finite difference time domain (FDTD) simulations. Hence, cavity-to-cavity distance and cavity size are indeed equivalent. Reflection spectra of moiré cavities with plasmonic cavity sizes ranging from 2.5 to $15.0 \mu \mathrm{m}$ with fixed J-aggregate strength and $40 \mathrm{~nm}$ thick metal films were simulated, and the corresponding dispersion curves were plotted in the form of heat maps. Rabi splitting energy, representing the strength of exciton-plasmon coupling was calculated from the dispersion curves. In a similar way, $Q$-factors were calculated from the dispersion curves. Here we present the results for cavity size of $2.5 \mu \mathrm{m}$ for comparison with the experiment. The $Q$-factor increases with the cavity size, while the Rabi splitting energy decreases with the $Q$-factor. A decrease of the Rabi splitting energy, while the cavity size increases is due to a decrease of the mode volume of the cavity. The Rabi splitting energy, $\hbar \Omega=2 g \sim 1 /(V)^{1 / 2}$ where $g$ is the coupling strength, is inversely proportional to the square root of the mode volume of the plasmonic cavity; hence, large enhancement of light matter interaction in small plasmonic cavities is expected [17,18]. In addition, in small cavities, the plasmonic mode confinement perpendicular to the surface might be another reason affecting stronger plasmon-exciton interaction in small cavities; see the Supporting Information. In a recent study, large plasmon-exciton coupling has been observed in small plasmonic nanocavities [18]. Furthermore, in another recent study, in gold nanowires, large Rabi splitting energies have been observed in small gold nanowires [19]. The 
dispersion curves of the bare moiré surfaces display a cavity mode due to cavity-confined SPPs. When bare PVA film was uniformly coated on top of the plasmonic cavities, the lower branch of the dispersion curve shows a redshift, which is confirmed by the theoretical simulation results. The energy of SPPs at the PVA-metal interface is smaller than the energy of SPPs at the air-metal interface because of the increase in effective refractive index [20]. Finally, a J-aggregate embedded PVA layer was placed on the metallic moiré surface. From Figs. 2(a) and 2(b), which are for the exciton-plasmon coupling on 2.5 and $5 \mu \mathrm{m}$ moiré surfaces, respectively, it can be inferred that the cavity mode is split into two new branches at approximately $590 \mathrm{~nm}$. Likewise, the same procedure was repeated for a $9 \mu \mathrm{m}$ moiré cavity. The $Q$-factors of cavities were calculated from the full width at half-maximum of the reflection spectrum at a single angle for the bare moiré surfaces; see Fig. 2(c). Rabi splitting energies were extracted from the dispersion curves of J-aggregate coated moiré surfaces; hence, the cavity size and Rabi splitting energy relations are given in Fig. 2(d).

In order to confirm our theoretical results, we performed spectral reflection measurements on moiré surfaces uniformly coated with a PVA layer containing J-aggregate dyes.

For the experiment, the samples were structured by using double exposure laser interference lithography. Then the fabricated coupled cavities were coated with $40 \mathrm{~nm}$ thick Ag film by thermal evaporation, and then the J-aggregate layer was spin-coated on top of the cavities. The polarization-dependent spectroscopic reflection measurements were performed in the well-known Kretschmann configuration. For different incidence angles, the cavities coated with J-aggregate dyes were scanned for a wavelength range. As a result, dispersion curves in the form of a heat map were obtained. The experimental results for the exciton-plasmon coupling on moiré surfaces with $2.5 \mu \mathrm{m}$ cavity size are shown in Fig. 3(a), which is quite similar to the simulation results for the $2.5 \mu \mathrm{m}$ moiré cavity; see Fig. 3(b). These results for $2.5 \mu \mathrm{m}$ moiré surfaces are in good agreement with the experimental data. Since we observed relative changes of Rabi splitting in this Letter, we indeed plotted the dispersion curves as wavelength versus angle and calculated the Rabi splitting energies from these plots. It should be noted that
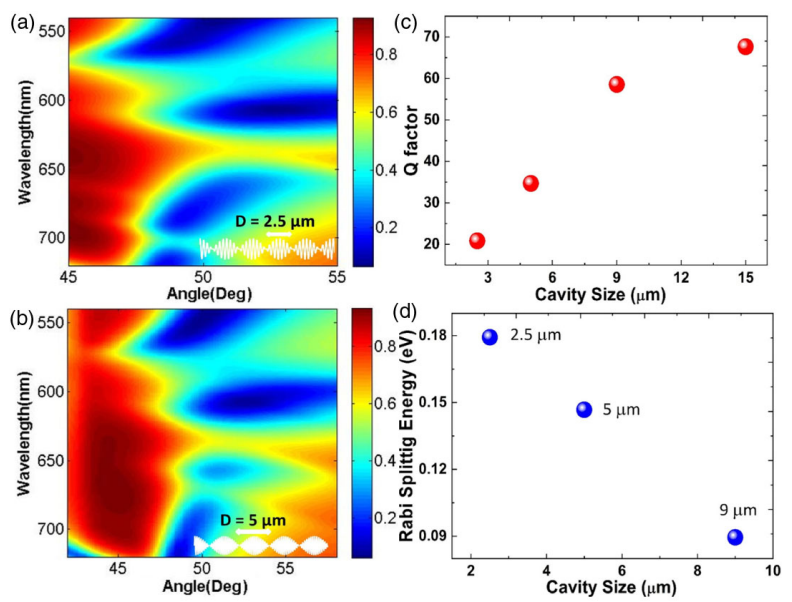

Fig. 2. Large exciton-plasmon coupling on small moiré surfaces. Exciton-plasmon coupling on (a) 2.5 and (b) $5 \mu \mathrm{m}$ cavity size moiré surfaces coated with J-aggregate dyes. (c) The $Q$-factor of the cavity increases with the size of the cavity. (d) Cavity size response of the Rabi splitting energy.

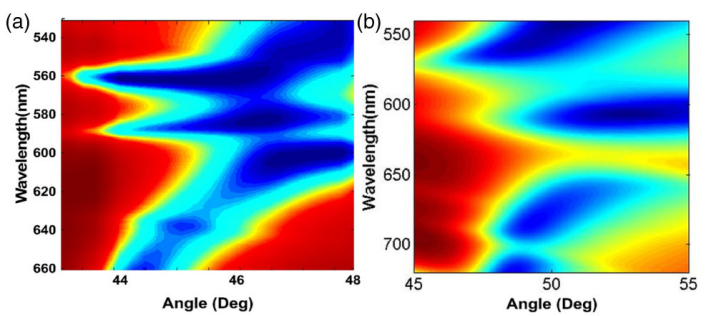

Fig. 3. (a) Experimental and (b) simulation results for $2.5 \mu \mathrm{m}$ moiré plasmonic cavity.

for a fixed incidence angle, the wavevector is $k=2 \pi / \lambda \sin (\theta)$ is not constant. Therefore, the magnitude of the Rabi splitting energy calculated from the wavelength versus angle dispersion curves and energy versus wave vector dispersion curves are not the same. These results for $2.5 \mu \mathrm{m}$ moiré surfaces are in good agreement with the experimental data.

FDTD calculations were conducted to study the effects of metal film thickness on exciton-plasmon coupling. Lorentz oscillator strength of the aggregated dye molecules was fixed, while Ag film thickness was varied from 20 to $50 \mathrm{~nm}$. The dispersion curves for each $\mathrm{Ag}$ film thickness were calculated in the Kretschmann configuration. Rabi splitting energies were calculated from the dispersion curves at the momentum where the energy splitting between the upper and lower branches reaches its minimum value. The results for J-aggregates on a flat Ag surface are shown in Fig. 4. There is a diffuse minimum in blue at the absorption wavelength, $590 \mathrm{~nm}$, for the $25 \mathrm{~nm}$ thick metal. For the $30 \mathrm{~nm}$ thickness, absorption of the J-aggregate disappears with a clear gap opening up indicating the onset of strong coupling. Increasing the metal film thickness $(t)$ results in an increase of Rabi splitting energy. For metal film thickness beyond $35 \mathrm{~nm}$, we observe that an increase in the Rabi splitting energy is accompanied with a decrease in intensity of the modes at the upper and lower polariton branches. The theoretically calculated Rabi splitting energies for various metal film thicknesses are shown in Fig. 4. The magnitude of the Rabi splitting energy rapidly increases for very thin metal films and increases linearly thereafter. For thick metal films, the Rabi splitting starts
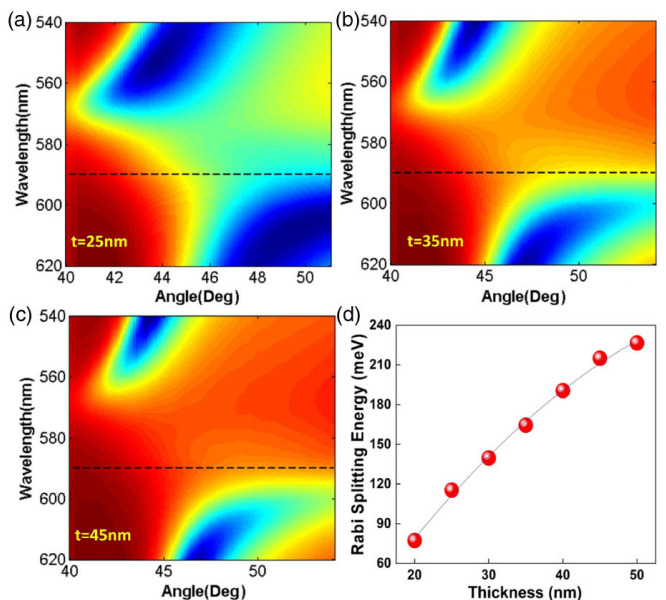

Fig. 4. Metal film thickness dependence of exciton-plasmon coupling. The dispersion curves for metal film thicknesses of (a) 25, (b) 35, and (c) $45 \mathrm{~nm}$ with a J-aggregate layer are shown. The dashed line is the absorption line of J-aggregate at $590 \mathrm{~nm}$. (d) Rabi splitting energy as a function of metal thin film thickness. 


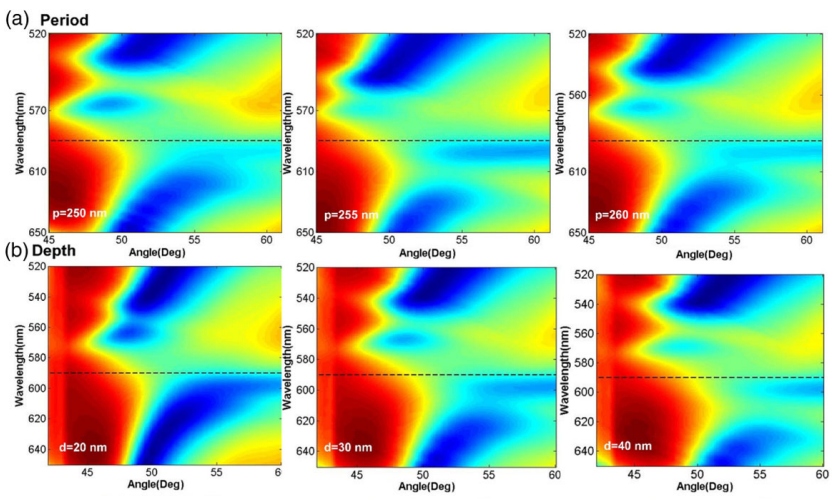

Fig. 5. Characterization of the effects of (a) the groove periodicity and (b) depth on the exciton-plasmon coupling on uniform gratings.

to decrease due to the fact that SPP modes are no longer supported. These results are consistent with the previously obtained experimental data and theoretical results [5].

The construction of a moire cavity requires the understanding of the exciton-plasmon coupling behavior on uniform gratings. The effect of grating periodicity on the excitonplasmon coupling on uniform gratings has been studied in detail and, the results are shown in Fig. 5. The periodicity of the uniform grating was varied from 245 to $300 \mathrm{~nm}$, while the grating depth and plasmonic layer thicknesses were kept constant. Varying the periodicity of the uniform grating tunes the spectral location of the plasmonic bandgap. If a J-aggregate layer is added on top of the uniform grating structure, depending on the relative position of the J-aggregate absorption peak with respect to the plasmonic bandgap, the band edge closest to the absorption line of the J-aggregate splits for grating periods greater than $260 \mathrm{~nm}$ and less than $250 \mathrm{~nm}$ that are not shown. However, for uniform gratings with the periodicity of $250 \mathrm{~nm}$, there occur splitting at both edges of the bandgap, Fig. 5(a). The density of the plasmonic states [21] is very low in the bandgap, but increases rapidly as the band edge is approached. When the absorption line of the J-aggregate is located at the plasmonic band edges, coupling of excitons with the plasmonic modes at the plasmonic band edges takes place; thus, new hybrid states occur at the band edges. Formation of these new states can be clearly seen in the simulations on gratings with periods 250 , 255, and $260 \mathrm{~nm}$, Fig. 5(a). When the periodicity of the grating is increased further, new hybrid states start to emerge. As shown in Fig. 5(a), the magnitude of the Rabi splitting energy is constant for nearly all of the periodicities of the uniform grating. In order to see the effect of the grating depth on the plasmon-exciton coupling, the depth of the uniform grating has been tuned from 20 to $50 \mathrm{~nm}$ in $10 \mathrm{~nm}$ steps. It should be noted here that, in our earlier work, we have demonstrated that group velocity of SPPs in plasmonic-coupled cavities can be tuned by varying the grating groove depth [22]. As shown in the Fig. 5(b), the Rabi splitting energy does not change with the grating groove depth.

In conclusion, we have extensively studied the excitonplasmon coupling in moiré type plasmonic cavities. The effects of various parameters such as periodicity, grating depth, metal film thickness, and cavity size, on exciton-plasmon coupling were extensively studied. Strong exciton-plasmon coupling was observed in several cases. Tuning the strength of excitonplasmon coupling with metal thin film thickness, grating depth, grating periodicity, or cavity size gives us the ability to control the dispersion diagrams. Enlarging moiré cavity size results in a high $Q$-factor, as it was previously demonstrated but Rabi splitting energy gets smaller. The calculated Rabi splitting energies for different cavity sizes range from 80 to $180 \mathrm{meV}$. Our results demonstrate that large Rabi splitting energies can be observed for small plasmonic cavities due to the small mode volume of the coupled small cavities. This level of control may make it possible to fabricate efficient and convenient plasmonic cavities for surface enhanced spectroscopy and nonlinear plasmonic applications leading to the demonstration of plasmonic lasers with cavity feedback [23].

Funding. Scientific and Technological Research Council of Turkey (110T790, 110T589).

Disclosures. The authors declare no conflicts of interest.

See Supplement 1 for supporting content.

\section{REFERENCES}

1. J. Bellessa, C. Symonds, K. Vynck, L. Beaur, A. Brioudec, and A. Lemaitre, Superlattices Microstruct. 49, 209 (2011).

2. C. Weisbuch, M. Nishioka, A. Ishikawa, and Y. Arakawa, Phys. Rev. Lett. 69, 3314 (1992).

3. M. A. Noginov, G. Zhu, M. Mayy, B. A. Ritzo, N. Noginova, and V. A. Podolskiy, Phys. Rev. Lett. 101, 226806 (2008).

4. I. Pockrand, A. Brillante, and D. Mobius, J. Chem. Phys. 77, 6289 (1982).

5. S. Balci, C. Kocabas, S. Ates, E. Karademir, O. Salihoglu, and A. Aydinli, Phys. Rev. B86, 235402 (2012).

6. A. V. Akimov, A. Mukherjee, C. L. Yu, D. E. Chang, A. S. Zibrov, P. R. Hemmer, H. Park, and M. D. Lukin, Nature 450, 402 (2007).

7. A. Trugler and U. Hohenester, Phys. Rev. B 77, 115403 (2008).

8. J. Bellessa, C. Bonnand, J. C. Plenet, and J. Mugnier, Phys. Rev. Lett. 93, 036404 (2004).

9. D. G. Lidzey, D. D. C. Bradley, A. Armitage, S. Walker, and M. S. Skolnick, Science 288, 1620 (2000).

10. K. J. Vahala, Nature 424, 839 (2003).

11. T. Yoshie, A. Scherer, J. Hendrickson, G. Khitrova, H. M. Gibbs, G. Rupper, C. Ell, O. B. Shchekin, and D. G. Deppe, Nature 432, 200 (2004).

12. F. Benz, M. K. Schmidt, A. Dreismann, R. Chikkaraddy, Y. Zhang, A. Demetriadou, C. Carnegie, H. Ohadi, B. de Nijs, R. Esteban, J. Aizpurua, and J. J. Baumberg, Science 354, 726 (2016).

13. S. Balci, Opt. Lett. 38, 4498 (2013).

14. D. G. Baranov, M. Wersall, J. Cuadra, T. J. Antosiewicz, and T. Shegai, ACS Photonics 5, 24 (2018).

15. A. Kocabas, S. S. Senlik, and A. Aydinli, Phys. Rev. Lett. 102, 063901 (2009).

16. S. Balci, M. Karabiyik, A. Kocabas, C. Kocabas, and A. Aydinli, Plasmonics 5, 429 (2010).

17. P. Torma and W. L. Barnes, Rep. Prog. Phys. 78, 013901 (2015).

18. C. M. Guvenc, F. M. Balci, S. Sarisozen, N. Polat, and S. Balci, J. Phys. Chem. C 124, 8334 (2020).

19. M. Kumar, J. Dey, M. S. Verma, and M. Chandra, Nanoscale 12, 11612 (2020).

20. W. L. Barnes, J. Opt. A 8, S87 (2006).

21. P. Nordlander and F. Le, Appl. Phys. B 84, 35 (2006).

22. S. Balci, A. Kocabas, C. Kocabas, and A. Aydinli, Appl. Phys. Lett. $\mathbf{9 7}$ 131103 (2010).

23. E. Karademir, S. Balci, C. Kocabas, and A. Aydinli, ACS Photonics 2, 805 (2015). 